\title{
Sur la nature des centres de sensibilité des emulsions photographiques
}

\author{
Par A. Hautot et H. Sauvenier \\ Laboratoire de Physique Générale de l'Université de Liège \\ (Z. Naturforschg. 6 a, 370-371 [1951]; eingegangen am 19. April 1951)
}

A Monsieur J. Eggert pour son 60ième anniversaire

\begin{abstract}
On avance que les centres de sensibilité dans les émulsions aux halogénures d'argent sont essentiellement des accidents de cristallisation superficiels ou internes. Ces centres sont créés en cours de maturation physique, la maturation chimique ayant pour rôle principal d'activer la formation des germes d'image latente dans ces centres. On donne deux séries de résultats expérimentaux qui paraissent s'accorder avec ce point de vue.
\end{abstract}

1.

$\mathrm{O}^{n}$ n considère généralement que l'existence des centres de sensibilité dans les grains de bromure d'argent de l'émulsion photographique est liée à l'action exercée sur les grains par les composés sulfurés de la gélatine et, en conséquence, que la plupart de ces centres sont constitués lors de la maturation chimique. Cependant, plus récemment, un autre point de vue s'est fait jour dans lequel le rôle de la maturation chimique serait plutôt d'activer les centres de sensibilité; dans ce cas, ceux-ci seraient déjà présents à la fin de la maturation physique.

\section{2.}

Afin de vérifier ce point important, on a étudié l'effet de la maturation chimique sur des émulsions préparées spécialement. Il s'agit d'émulsions ammoniacales préparées avec la même gélatine et en présence d'un excès de $\mathrm{KBr}$ de $10 \%$, dans des conditions variées de précipitation et de maturation physique. Sur le graphique inférieur de la figure 1, on a classé les 16 émulsions étudiées par ordre de sensibilité croissante; la sensibilité est mesurée par l'inertie. Aucune des émulsions auxquelles se rapporte le graphique inférieur n' subi la maturation chimique.

Le graphique supérieur concerne les mêmes émulsions ayant subi la maturation chimique optimum (= arrêtée à l'apparition du voile) à l'aide d'une même gélatine.

Le graphique intermédiaire se rapporte aux mêmes émulsions ayant subi une maturation chimique de durée égale au tiers de la durée optimum.

On constate que la maturation chimique multiplie la sensibilité par un facteur qui ne diffère pas fortement d'une émulsion à l'autre. En désignant par $S_{0}$ la sensibilité de l'émulsion non mûrie chimiquement et par $S_{1}$ celle de l'émulsion mûrie chimiquement, et en calculant le rapport $S_{1} / S_{0}$ pour 15 émulsions (la 1ère émulsion à grain très fin est difficilement reproductible et n'a pas été incluse dans le calcul), on trouve, comme valeur moyenne,

$$
\frac{S_{1}}{S_{0}}=2.21 \pm 0.46
$$

pour les émulsions ayant subi la maturation chimique optimum; et

$$
\frac{S_{1}}{S_{0}}=1.44 \pm 0.21
$$

pour celles qui ont subi la maturation chimique partielle. Ces nombres, qui diffèrent certainement pour d'autres classes d'émulsions, montrent que la matu-

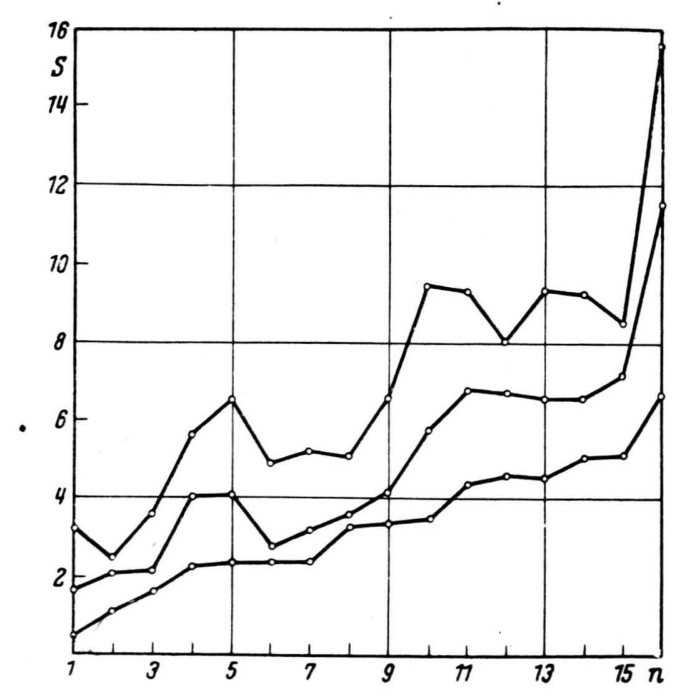

Fig. 1. Variation de la sensibilité de diverses émulsions ammoniacales avec la durée de la maturation chimique. 
ration chimique sensibilise à peu près de la même manière des émulsions de sensibilités initiales diverses; on peut donc conclure que la maturation rend les centres de sensibilité plus opérants mais qu'elle ne les crée pas.

D'autres expériences conduisent à la même conclusion. Lorsqu'on irradie une émulsion de manière à produire une image solarisée, chaque grain contient de $10^{4}$ à $10^{7}$ atomes d'argent, selon qu'on arrête l'exposition au début de la solarisation ou du second renversement; avec une telle exposition, la plupart des centres de sensibilité contiennent un germe d'image (ou en ont contenu un avant de solariser). Quel que soit le mécanisme de la solarisation (rehalogénation ou défaut catalytique des germes), son importance dépend étroitement du nombre de germes superficiels par grain, donc aussi du nombre de centres de sensibilité superficiels. La comparaison de l'importance de la solarisation avant et après la maturation chimique permet donc de juger si le nombre moyen de centres de sensibilité superficiels par grain a été modifié par la maturation chimique. Des expériences faites sur ce sujet par $\mathrm{S}$ a u v e n i e $\mathrm{r}^{1}$ révèlent bien de légères différences dans l'importance de la solarisation avant et après la maturation chimique mais permettent cependant d'affirmer que le caractère solarisable ou non solarisable d'une émulsion est fixé, pour une part considérable, à la fin de la maturation physique. Le tableau suivant valide cette conclusion: dans la ligne supérieure figure le rapport de solarisation $\frac{\Delta-\delta}{\Delta}$ pour l'émulsion non mûrie chimiquement et dans la ligne inférieure, le même rapport pour l'émulsion ayant subi la maturation optimum; le tableau concerne 15 émulsions ammoniacales différentes au bromure d'argent; $\Delta$ réprésente la densité maximum (au début de la solarisation) et $\delta$ la densité minimum au seuil du second renversement ${ }^{2}$.

\begin{tabular}{|c|c|c|c|c|c|c|c|}
\hline $0,16_{5}$ & 0,30 & 0,75 & 0,88 & 0,44 & 0,5 & 0,6 & 0,72 \\
\hline 0,10 & 0,42 & 0,92 & 0,87 & 0,42 & 0,5 & 0,6 & 0,73 \\
\hline 0,7 & & $79_{5}$ & 0,84 & 0,84 & 0,58 & 0,78 & 0,87 \\
\hline $0,7 \mathrm{~s}$ & & 75 & 0,85 & 0,76 & 0,62 & 0,82 & 0,90 \\
\hline
\end{tabular}

1 H. S a uve n i er, Sci. Ind. photogr. 19, 13 [1948].

2 Le rapport $\frac{\Delta-\delta}{\Delta}$ a été proposé pour mesurer l'importance de la solarisation (cf. A. Ha u t o t et H. S a u ve n i e r, Sci. Ind. photogr. 19, 281 [1948].
Il paraît donc prouvé que l'importance de la solarisation ne dépend que très peu de la maturation chimique et, par conséquent, que la plupart des centres de sensibilité sont présents dans les grains à la fin de la maturation physique.

\section{4.}

Ces faits remettent en cause la nature même des centres de sensibilité. Ceux-cí, étant présents à la fin de la maturation physique, doivent être constitués principalement, et peut-être exclusivement, par des accidents cristallographiques superficiels, tels que des pointes, des arêtes ou des trous à la surface des grains. De tels accidents géométriques peuvent exister aussi dans l'intérieur des grains, sous la forme de failles, de glissements, de tensions ou de surfaces de séparation entre les divers microcristaux dont un grain peut être constitué: ce dernier type de centre de sensibilité interne doit être particulièrement fréquent dans les gros grains formés par le processus de coalescence.

Assurément, la maturation physique s'accompagne déjà d'une certaine maturation chimique puisque la gélatine employée lors de la maturation physique est déjà généralement d'un type quelque peu actif. Cependant, vu la croissance relativement rapide des grains (en moyenne, un plan réticulaire est édifié en 1 seconde dans les conditions usuelles de maturation physique), la maturation chimique qui accompagne inévitablement la maturation physique a une durée relativement courte puisqu'elle affecte seulement les couches superficielles des grains et que, après plusieurs secondes, celles-ci sont devenues internes. On ne pourrait donc guère attribuer à cette maturation chimique relativement très courte la création des centres de sensibilité. Il est donc plausible d'admettre que c'est la croissance irrégulière des grains dans la solution qui détermine principalement l'existence des centres des sensibilité.

L'accroissement ultérieur de la sensibilité des grains sous l'effet de la maturation chimique peut lui-même être attribué à deux causes; la formation dans les centres de sensibilité préexistants d'agrégats formés d'électrons et d'ions interstitiels $\mathrm{Ag}^{+}$, un tel agrégat jouant le rôle de sousgerme au moment de l'exposition lumineuse (hypothèse de Mitchell) et l'accroissement du nombre ou de la mobilité des particules du réseau (défauts de $\mathrm{Schottky}$ ou de $\mathrm{Frenkel}$ ) dont le transfert dans les centres de sensibilité concourt à former les germes d'image latente. 